\title{
Diffuse neural coupling mediates complex network dynamics through the formation of quasi-critical brain states
}

\section{Authors}

Eli J. Müller ${ }^{*}$, Brandon Munn ${ }^{1}$ and James M. Shine ${ }^{1,2}$

\section{Affiliations}

1 Brain and Mind Centre, The University of Sydney, Sydney, NSW, Australia

2 Complex Systems Research Group, The University of Sydney, Sydney, NSW, Australia

\section{Corresponding author:}

* Eli J. Müller - eli.muller@sydney.edu.au 


\section{Abstract}

The biological mechanisms that allow the brain to balance flexibility and integration remain poorly understood. A potential solution to this mystery may lie in a unique aspect of neurobiology, which is that numerous brain systems contain diffuse synaptic connectivity. In this manuscript, we demonstrate that increasing diffuse cortical coupling within a validated biophysical corticothalamic model traverses the system through a quasi-critical regime in which spatial heterogeneities in input noise support transient critical dynamics in distributed sub-regions. We then demonstrate that the presence of quasi-critical states coincides with known signatures of complex, adaptive brain network dynamics. Finally, we demonstrate the presence of similar dynamic signatures in empirical whole brain human neuroimaging data. Together, our results establish that modulating the balance between local and diffuse synaptic coupling in a thalamocortical model subtends the emergence of quasi-critical brain states that act to flexibly transition the brain between unique modes of information processing. 


\section{Introduction}

The brain is a complex, adaptive system that is organized across multiple spatial and temporal scales. Systems arranged in this way must solve a number of competing challenges. First, they must balance segregation - the need to retain precise, specialist functional capacities - and integration - in which information from segregated sub-regions is recombined at larger spatiotemporal scales (Shine et al., 2019a; Sporns et al., 2000). Second, the brain must remain flexible enough to retain sufficient sensitivity to fluctuations in evolving fitness landscapes (Kehagia et al., 2010). Finally, the systems must coordinate these capacities in ways that are energetically frugal (Bullmore and Sporns, 2012), which favours systems with relatively low-dimensional architectures (Cunningham and $\mathrm{Yu}, 2014$ ). How the brain is arranged to achieve these distinct constraints, and what physical mechanisms underpin them, remains poorly understood.

A solution to this challenge may be found in a somewhat over-looked principle of neuroanatomy. A number of circuits in the brain, such as the ascending neuromodulatory system (Edlow et al., 2012) and the non-specific, 'matrix' cells of the thalamus (Jones, 2002), project their axons in a relatively diffuse pattern that targets multiple distinct neural regions. These circuits are incompatible with the traditional notion of 'message passing' between individual neurons that is typically ascribed to targeted, feed-forward projections between neurons (Aertsen et al., 1996). So why might these highly conserved, diffuse connections exist as such a prominent feature of neuroanatomy?

A potential benefit of balancing targeted and diffuse coupling is that systems structured in this way may be able to support multiple distinct modes of processing. For instance, targeted connections between neural sub-regions will influence local neighbours in a relatively segregated mode, whereas diffuse connections may force distant regions into novel regimes that are impacted more strongly by the global brain state. Crucially, by modulating the amount of global, diffuse connectivity, the system could control its information processing capacity .

Systems that support multiple distinct modes often exhibit optimal functional properties at the transition point (or critical point), such as maximizing information transmission, the dynamic range, and the number of metastable states (Deco and Jirsa, 2012; Muñoz, 2018). Rather than balancing precisely at a specific critical point, there is now robust evidence to suggest that complex systems such as the brain may 
display an enlarged/stretched critical point. This stretched critical regime (quasicritical) allows the system to more readily utilise the optimal functional properties bestowed at criticality (Cocchi et al., 2017; Tagliazucchi et al., 2012; Williams-García et al., 2014; Wilting and Priesemann, 2018). Near this quasi-critical region of state space, heterogeneity within the brain should allow sub-regions to experience transient excursions into the quasi-critical regime (Moretti and Muñoz, 2013; Vojta, 2006). This would allow the system to harness the benefits of criticality (e.g., divergence of correlation length), without the associated risk of transitioning en masse into a pathological state of global synchronization (Breakspear et al., 2006; Müller et al., 2017).

In this manuscript, we propose that this mechanism could be exploited in the brain by modulating the balance between local and diffuse synaptic coupling in the thalamocortical system. This in turn would imbue the system with the capacity to support the complex, adaptive system dynamics that support higher brain function.

\section{Results}

To test the hypothesis that diffuse coupling promotes a diversity of quasi-critical neural states, a network of biophysically-plausible corticothalamic neural mass models was used to simulate large-scale human brain activity (Fig. 1). Neural mass models, which are a spatially discretised class of a neural field model, provide a tractable framework for the analysis of large-scale neuronal dynamics by averaging microscopic structure and activity (Jirsa and Haken, 1996; Nunez, 1974; Robinson et al., 1997, 1998; Wilson and Cowan, 1973; Wright and Liley, 1996). These models are flexible, physiologically realistic and inherently non-linear, (Deco et al., 2008; Rennie et al., 1999; Robinson et al., 1997, 1998, 2001, 2002, 2005; Wilson and Cowan, 1973; Wright and Liley, 1996), and have successfully accounted for many characteristic states of brain activity (Bojak and Liley, 2005; Breakspear et al., 2006; Deco et al., 2008; Jirsa and Haken, 1996; Roberts and Robinson, 2008; Robinson et al., 2002; Steyn-Ross et al., 2004; Wilson and Cowan, 1973). Importantly, this work extends an existing and validated biophysical model, which itself has been extensively constrained by human electrophysiology data (Abeysuriya et al., 2015). This feature ensures that we have oriented the system to a plausible region of state space, and further implies that our results will lead to testable empirical predictions related to the impact of diffuse inputs. 
The specific neural mass model used in our study contained four distinct neural populations: an excitatory pyramidal cell, $e$, and an inhibitory interneuron, $i$, population in the cortex; and an excitatory specific relay nuclei, $s$, and inhibitory thalamic reticular nuclei, $r$, population in the thalamus. The parameters from the model were fit to a region of state-space defined by the awake, human brain using field potentials from human scalp EEG data (Abeysuriya et al., 2015; Robinson et al., 2004). We simulated a 12×12 network of corticothalamic neural masses (Fig. 1a) using the neural field simulation software, nftSim (Sanz-Leon et al., 2018). The parameters for each neural mass were identically set to "eyes-closed" estimates (Abeysuriya et al., 2015), which results in simulated activity with a characteristic $1 / f$ spectrum and a peak in the alpha frequency band $(8-13 \mathrm{~Hz})$.

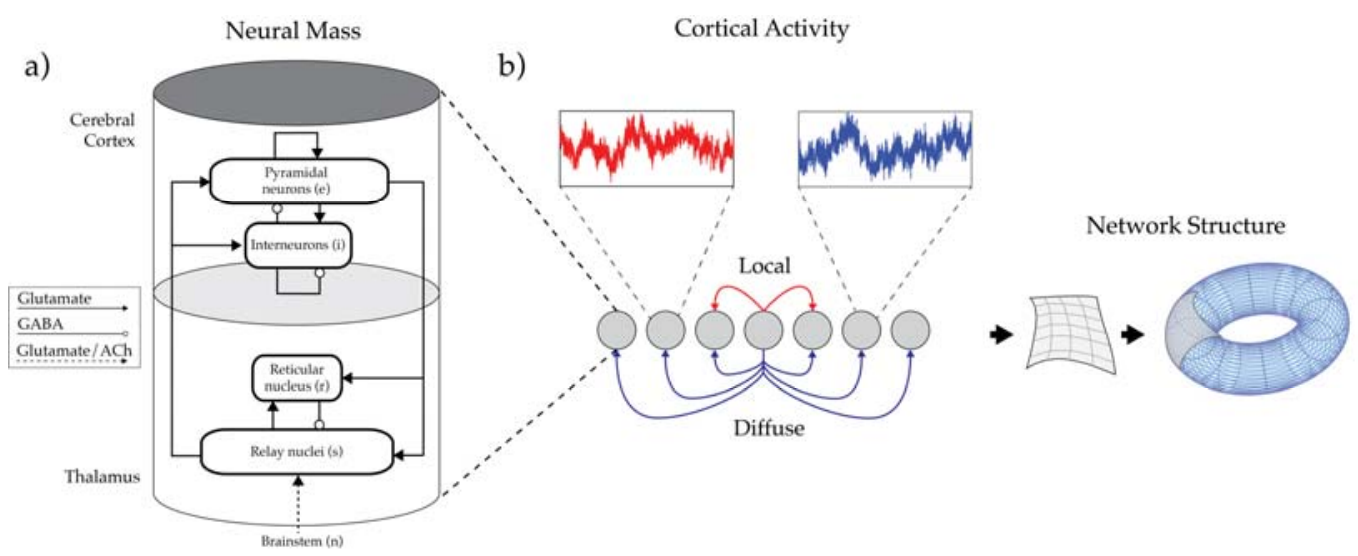

c)

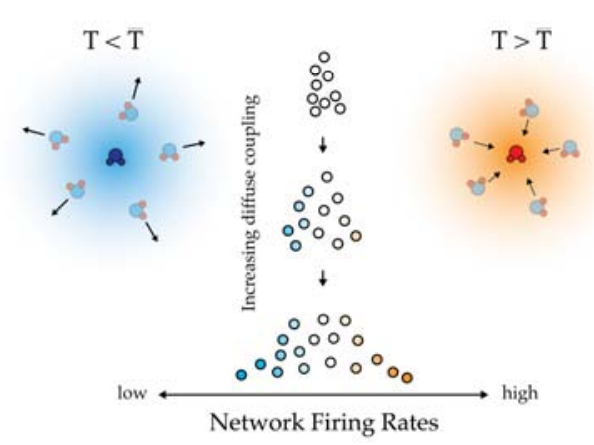

d)

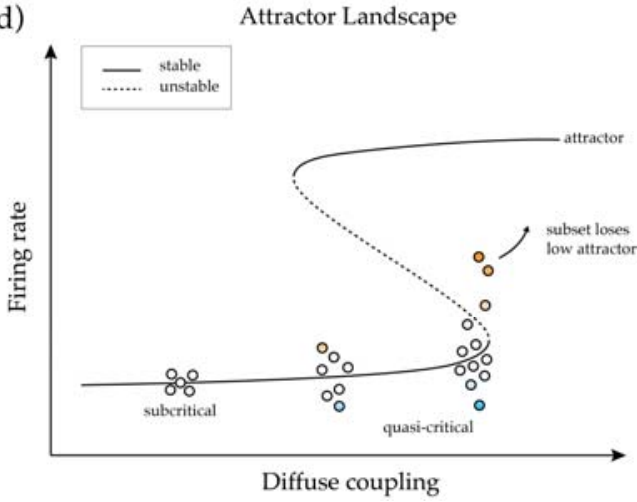

Figure 1 - Model schema. (a) Corticothalamic neural mass model implemented at each node of the network: each mass was comprised of four distinct cellular populations: an excitatory cortical pyramidal cell (' $e$ '), an inhibitory cortical interneuron (' $i$ '), an excitatory, specific thalamic relay nucleus ('s'), and an inhibitory thalamic reticular nucleus (' $r$ '), with intra-node corticothalamic neural mass coupling defined according to known anatomical connectivity; (b) Connectivity schematic local and diffuse coupling with periodic boundary conditions (toroidal topology); (c) Distribution of 
nodal firing rates across the network - an increase in diffuse coupling subsequently increases the standard deviation of firing rates, with the tails of this distribution having greater above (and below) average values. (d) Qualitative effect of increasing diffuse coupling in the presence of heterogeneity on the attractor landscape: increased diffuse coupling shifts all nodes towards their local saddle-node bifurcation point. In the middle of this continuum, the heterogeneous inputs allow a particular subset of nodes (shaded orange) to cross this point and the activity of these nodes begins to move towards the high firing attractor.

In addition to the identical intra-node coupling, our model contained two classes of connectivity: local coupling, which was defined as a connection between an excitatory population in the cortex and its immediate neighbours (with diagonal nodes additionally scaled by a spatial decay factor of $1 / \sqrt{2}$ ); and diffuse coupling, which connected the pyramidal $e$ populations' activity to all other nodes in the network (Fig. 1b). The diffuse coupling term, which is defined as $\chi$, was swept through a range and was the only parameter changed in this work. Periodic boundary conditions (i.e., a toroidal topological structure) were applied so that each node had an equal number of local afferent connections.

The presence of structural heterogeneities in neural network models, such as the human connectome and neural networks in the Caenorhabditis elegans, have been shown to extend an idealized critical point into a region of state space that is known in statistical mechanics as a 'Griffiths phase' (Moretti and Muñoz, 2013; Vojta, 2006). This form of quasi-criticality is analogous to the inherent balance present between the liquid and gaseous phases of water at room temperature (Fig. 1c), during which time the vast majority of the water molecules are in their liquid phase. As the temperature rises towards water's boiling point, a subset of these molecules may, for a short time, collide with other energetic molecules in their immediate surroundings. From the vantage point of this subset, it would appear as though the temperature of the entire fluid had risen. Those regions with slightly more energy than others would be able to cross their own locally-defined bifurcation (or critical boundary) i.e., 'transition' into water vapour - while leaving the rest of the water molecules in their liquid phase. This phenomenon will occur more often as the temperature approaches the boiling point.

The brain may exploit a similar physical mechanism, whereby subregions cross locally-defined critical boundaries while the bulk of the global brain state remains subcritical. We hypothesized that the prevalence of these critical regions should be modulated by diffuse inputs, in a manner analogous to increasing temperature in 
the fluid to gas transition. In other words, increasing diffuse coupling in the brain could drive the system such that a subset of nodes can cross their locally-defined critical boundaries (e.g., orange nodes in Fig. 1d), while ensuring that the rest of the network remains in a sub-critical state (e.g., blue nodes in Fig. 1d). It is important to note that this phenomenon only occurs when there are heterogeneities within the system. In the model used here, only the simplest form of spatial heterogeneity was included: namely, an independent white noise drive (uniquely sampled from an identical Gaussian distribution) to each neural mass in the network. Based on these factors, we hypothesized that the combination of heterogeneity and elevated diffuse coupling, $\chi$, would be sufficient to transition a subset of nodes over their locallydefined bifurcation, which in turn should alter the information processing dynamics of the brain. In order to test this hypothesis, we needed to identify a way to track transient, super-critical excursions at the nodal level in our model.

\section{Quantifying regional dynamics through distance to local bifurcation}

In dynamical systems, such as the brain, activity is often defined by the systems' "attractors", which are idealized states that a system evolves towards under a wide variety of starting conditions (Miller, 2016). Multi-stable systems are those with more than one attractor present for a single set of parameters: each attractor has unique stability properties and can be explored by the system given appropriate inputs and/or initial conditions. The biophysical model utilized in this study describes a multi-stable system near a Hopf and a saddle-node bifurcation, both of which occur when a smooth incremental change in a control parameter (in our case, diffuse coupling) causes a qualitatively abrupt changes in the systems behaviour.

Knowledge of a nodes attractors is important for understanding the nodes behaviour, however it can be challenging to extrapolate patterns from local nodes to the activity of the whole network. This makes it difficult to define the presence (or absence) of quasi-critical brain state dynamics in large-scale network models. To solve this problem, we note that the bifurcation point for each corticothalamic neural mass can be identified as a function of a constant postsynaptic potential induced by incident activity from other nodes. Time independent solutions can then be produced by sweeping over this induced potential change in order to find the neural mass' bifurcation point (i.e., the point where the two low-firing attractors meet and annihilate each other, leaving only a stable high firing attractor). Furthermore, the time-independent solutions can be used to determine the linear response gains between each population within the neural mass (Fig. S2). 
As $\chi$ is increased, individual nodes become increasingly sensitive to their own inputs - that is, they have heightened 'response gain' (David Servan-Schreiber, 1990; Shine, 2019; Shine et al., 2018). This effect is characterized by a sigmoidal function that maps population average membrane potential to firing rate (Wilson and Cowan, 1973), as well as the slope (first derivative) of this function (Fig. S1). All of the simulated data in our experiment lies on the left-hand side of the peak in the gain curve (subpanel in Fig. S1), such that incremental increases in response gain have large effects on the nodes' activity (i.e., the slope of the function is positive; orange in the right side of Fig. S1), and hence, cause the region to cross its locally-defined bifurcation (Fig. 1d).

\section{Increasing diffuse coupling promotes quasi-critical states}

Armed with this approach, at each simulation time point, inputs to a given node can be translated into an instantaneous distance to the receiving nodes' bifurcation point. In this way, the strength of each nodes' attractor can be quantified, and the duration of excursions across the point where the attractor is no longer present during simulation can be accurately quantified. Here, the percentage of nodes that have crossed their local bifurcation is defined as $P_{c}$. As predicted by our hypothesis, increasing the amount of diffuse network coupling caused a non-linear increase in $P_{c}$ (Fig. 2). Based on the network-level activity patterns across $\chi$, we defined three 'working zones': a stable, subcritical zone $\left(\chi<1.20 \times 10^{-4} \mathrm{mV} \cdot \mathrm{s}\right.$; blue in Fig. 2), where $P_{c}=0$; a quasi-critical zone $\left(1.20<\chi<1.27 \times 10^{-4} \mathrm{mV} \cdot \mathrm{s}\right.$; green in Fig. 2), where $0<P_{c}<100$; and a saturated zone $\left(\chi>1.27 \times 10^{-4} \mathrm{mV} \cdot \mathrm{s}\right.$; red in Fig. 2), where $P_{c}=100$ in the second half of the simulation.

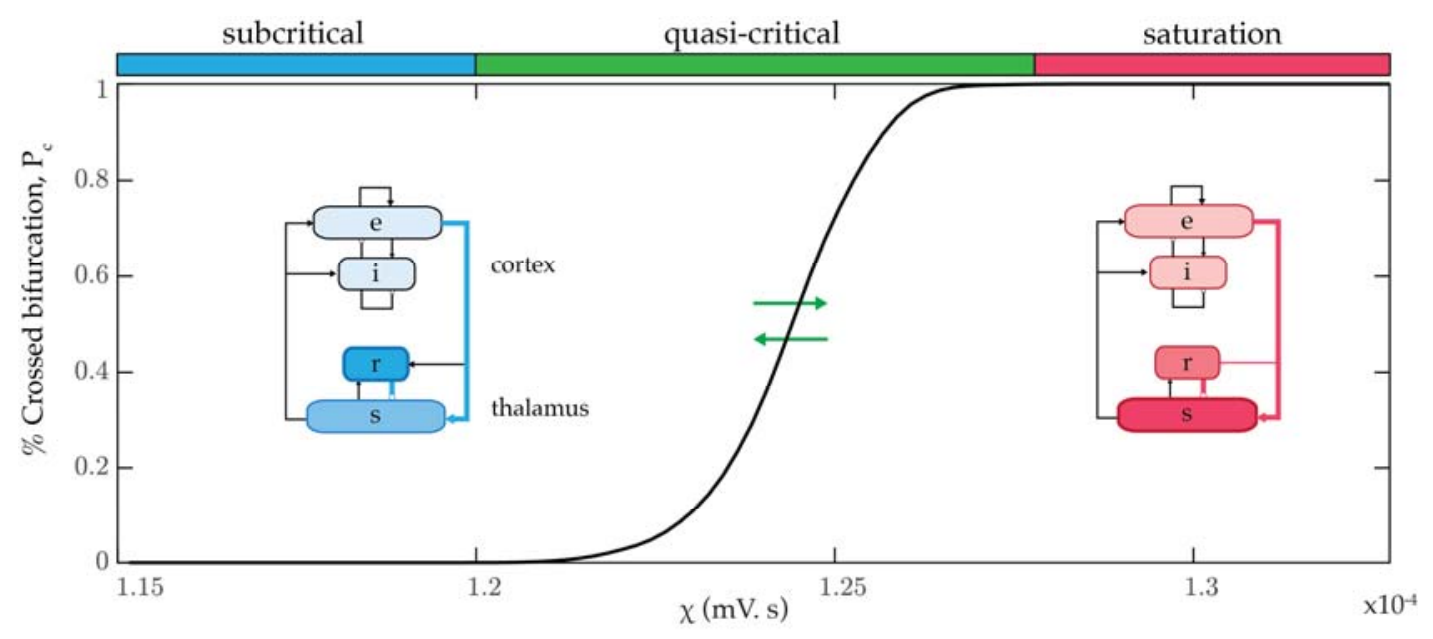


Figure 2 - Promoting quasi-critical states. (a) The time averaged percentage of nodes that have crossed their bifurcation, $P_{c}$, as a function of diffuse coupling, $\chi$. We identified three qualitative zones: a low variability subcritical zone (blue), where no nodes crossed their bifurcation point for the full duration of the simulation, a highly variable, quasi-critical zone (green) where at least one node was below its bifurcation during the second half of the simulation, and a saturated, oscillatory zone (red). The insets show steady state firing rates for each population within the neural mass model is represented via colour intensity.

Another benefit of neural mass models over more abstract approaches (such as the Kuramoto or Fitzhugh-Nagumo model; (Breakspear, 2017) is their superior physical interpretability. Each parameter within the neural mass is, in principal, a measurable biophysical quantity. We leveraged this feature to identify the relative firing rate of each neural population in our model. The three zones in our model were associated with qualitatively distinct steady state firing rate attractors for each population within the corticothalamic neural mass (inset of Fig. 2). Of note, the subcritical zone was associated with a higher firing rate in the $r$ thalamic population relative to the $s$ population (i.e., relative thalamic inhibition), whereas this relationship is inverted in the saturation zone (i.e., relative thalamic excitation). By construction, the quasicritical zone necessarily supports a mix of these two states, with the balance dictated non-linearly by the value of $P_{c}$ (Fig. 2). These results suggest that increasing diffuse coupling to the cortex had the effect of releasing a subset of excitatory thalamic $s$ neurons from inhibition, which in turn was reflected by the crossing of their local bifurcation point (Fig. S3).

We also observed qualitatively distinct effects at the whole-network level. The average regional correlations within each zone are displayed as a force-directed graph in Fig. 3a. The subcritical zone is dominated by local coupling and the saturation zone by diffuse coupling. Notably, the quasi-critical zone shows a mix of both these integrated and segregated topological states, and their coincidence is predicated on heterogeneity within the network. Somewhat trivially, if this heterogeneity is removed and diffuse coupling is increased, the entire network will cross the bifurcation point together with $P_{c}$ either $0 \%$ or 100 , which is equivalent to an isolated neural mass receiving increasing drive. In other words, confirming our hypothesis, the presence of the quasi-critical regime was due entirely to the presence of spatial heterogeneity and increasing the diffuse coupling term, $\chi$. 
a)

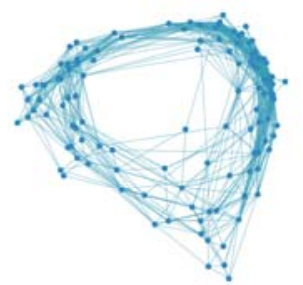

subcritical

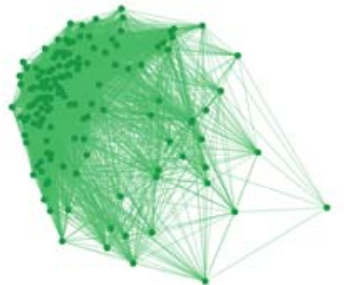

quasi-critical

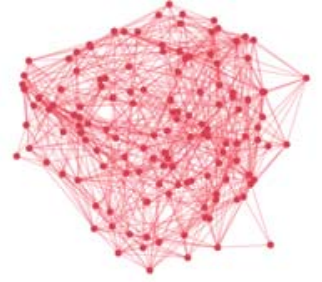

saturation

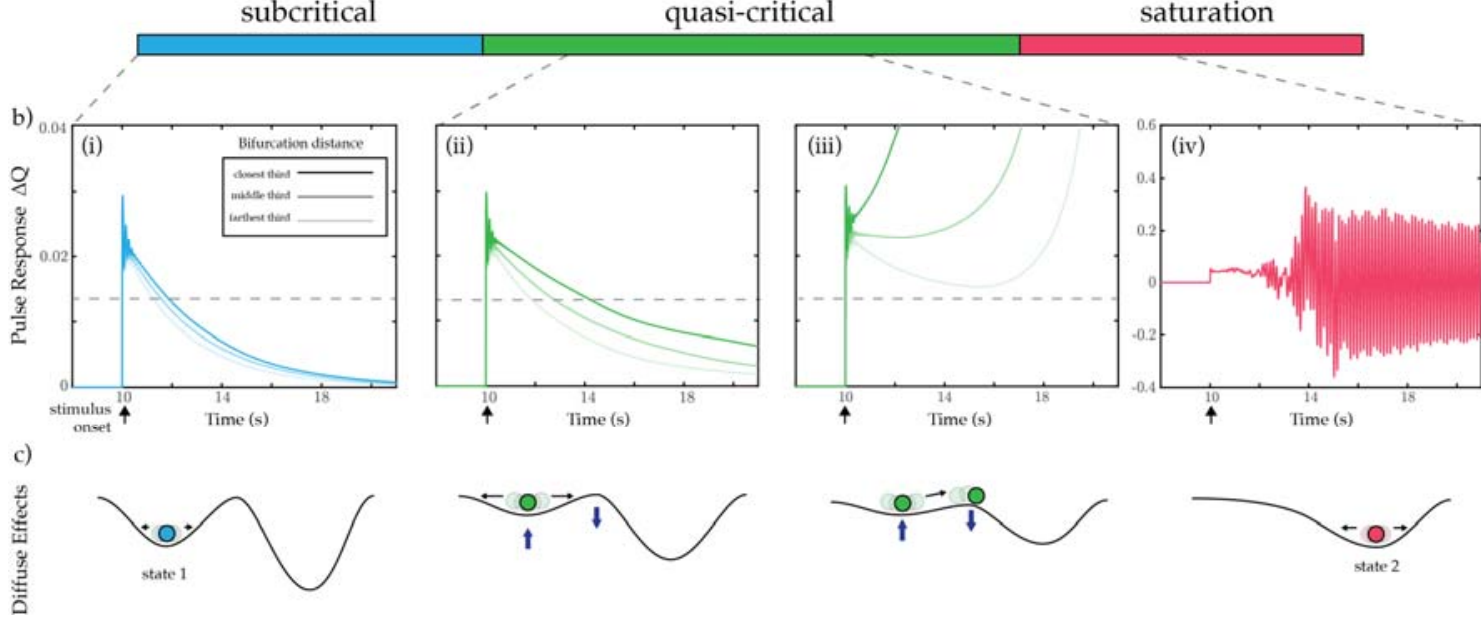

Figure 3 - Properties of quasi-critical states. (a) Average regional correlations within each zone shown as a force-directed graph; (b) For a given $\chi$, each node was stimulated with an excitatory rectangular pulse (amplitude $=1 \mathrm{mV}$; width $=10 \mathrm{~ms}$ ) at $\mathrm{t}=10 \mathrm{~s}$. The target nodes activity was then compared to simulated activity in the absence of the pulse using the same noise sequence in order to quantify the perturbation induced. The pulse results are sorted based on their mean distance to bifurcation in the preceding 8 time points. For visualization purposes we then average the activity within the closest, middle, and farthest thirds based on this sorted distance, and low-pass filter with a passband frequency of $0.001 \mathrm{~Hz}$. (i) $\chi=1.15 \times 10^{-4}$ (ii) $\chi=1.21 \times 10^{-4}$ (iii) $\chi=1.25 \times 10^{-4}$ (iv) $\chi=1.3 \times 10^{-4} \mathrm{mV} . \mathrm{s}$; Note the vertical axis on (iv) differs from (i) - (iii); (c) Qualitative effect of increasing diffuse coupling on the attractor landscape: in the sub-critical zone, the system was enslaved to the lower attractor; increasing $\chi$ into the quasi-critical zone had the effect of flattening the attractor landscape, allowing noise-driven excursions to transition nodes across their local bifurcation point; at high values of $\chi$, the system became enslaved to the higher attractor.

Based on previous literature (Fontenele et al., 2018; Moretti and Muñoz, 2013; Williams-García et al., 2014), we hypothesized that the quasi-critical regime should augment the network's sensitivity to incoming stimuli. To test this hypothesis, a series of network simulations were run wherein an excitatory pulse stimulus was applied separately to each node across several diffuse coupling values (see Fig. 3b: panels i-iv). For visualization purposes, the nodes were grouped based on their average distance to bifurcation in a brief window $(40 \mathrm{~ms})$ preceding stimulus. In line with other critical phenomenon, the response duration and sensitivity of the network increases with diffuse coupling as the system as a whole becomes more 
critical. This is equivalent to the lower attractor 'flattening', which in turn allows a greater proportion of individual nodes to transition onto the higher attractor (Fig. 3c).

In addition to the increased network sensitivity, flexibility is increased within the quasi-critical zone, with a greater spread of stimulus response durations observed (contrast Figs. 3b (i) and (ii)). This highlights the fact that within the quasi-critical zone, the dynamic repertoire is extended (Kinouchi and Copelli, 2006) and could provide a mechanistic description of the hierarchy of timescales inferred empirically (Cocchi et al., 2016; Honey et al., 2012). Together, these results demonstrate that increasing diffuse coupling transitions the network into a sensitive and complex state, which would likely be further enriched by the known spatial heterogeneity imbued by the white-matter of the structural connectome (Bullmore and Sporns, 2012).

\section{Network signatures of quasi-criticality}

When analysing empirical neuroimaging data, it is not possible to obtain direct evidence of a nodes' gain, nor it's distance from its' own bifurcation. Instead the putative signatures of complex, adaptive system dynamics must be estimated indirectly from empirical neuroimaging data (McIntosh and Jirsa, 2019). Here, we demonstrate that a number of these analytic measures show qualitative changes as a function of $\chi$, and thus together provide empirically accessible signatures of complex, adaptive dynamics (Fig. 4). For instance, the mean participation coefficient, which quantifies the extent of cross-community integration across the brain network (Shine et al., 2016), was low, yet regionally variable, in the sub-critical zone, rose sharply in the quasi-critical zone, and reached a ceiling in the saturated zone (Fig. 4a). This pattern is consistent with previous neuroimaging work that showed an increase in integration as function of cognitive task performance (Cohen and D’Esposito, 2016; Hearne et al., 2017; Shine et al., 2016).

Time-series variability (Fig. 4b; black) showed a similar monotonic increase with $\chi$, though with a more protracted course than network integration. In contrast, regional diversity (Fig. 4b; orange) initially increased before dropping and wavering in the quasi-critical zone, and ultimately increasing to its highest value in the saturated zone. Interestingly, an increase of time-series variability within the quasi-critical zone was preceded by two peaks in regional diversity, which was defined as the variance in the upper triangle of the region-wise functional connectivity matrix. In 
other words, a promotion of unique functional architectures across the network occurs within the quasi-critical zone and these appear distinct from an increase in sustained local variability.
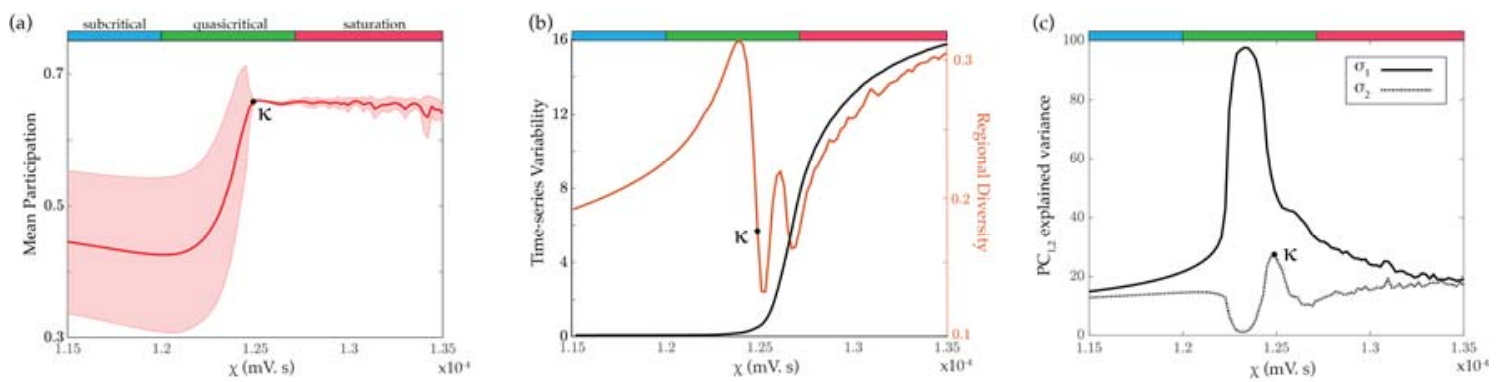

Figure 4 - Network topology and dimensionality. (a) Mean participation - which quantifies the extent to which a region functionally connects across multiple modules (these were calculated using a weighted version of the Louvain algorithm across all simulations); (b: left) Average time-series variability; (b: right) Regional diversity - defined as the variance in the upper triangle of the regionwise functional connectivity matrix; (c) $\mathrm{PC}_{1,2}$ explained variance. $\kappa$ demarcates corresponding points in all panels.

In previous work, we analysed human fMRI data to show that the brain reconfigures into a low-dimensional brain state across a diverse array of cognitive tasks (Shine et al., 2019). Similar patterns were observed here in the simulated data (Fig. 4c). Specifically, the percentage of variance explained by the first two principal components of the firing rate time series peaked in the quasi-critical zone, with the second principal component rising in explanatory power at a higher level of $\chi$ (Fig. 4c). Interestingly, the peak in the variance explained by the second principal component coincided with the peak in integration (corresponding to $\kappa$ in Fig. 4), and the peak in variance explained by the first principal component coincided with the first peak in regional diversity. Together, these results suggest that the quasi-critical zone is associated with an integrated, flexible and relatively low dimensional network architecture, which is consistent with recent empirical whole-brain imaging results (Shine et al., 2019a) and has implications for the information processing capacities of both artificial and biological networks.

\section{Orienting task and rest states from human fMRI data}

We were next interested in whether the complex, adaptive network signatures identified in our neural mass model would translate into differences in empirical, whole brain neuroimaging data. Based on previous work (Shine et al., 2016, 2019a) and the results of our biophysical model, we hypothesized that the network-wide 
effects of distinct cognitive states would be dissociable based on the measures that were found to be have unique signatures in the quasi-critical zone. Specifically, we predicted that task performance should be associated with increased diffuse network coupling, reflective of increased ascending arousal neuromodulation (Shine et al., 2018) and increased thalamic engagement (Shine et al., 2019b), thus allowing information from functionally specialized regions, optimally formed in the segregated state, to be integrated across broad spatiotemporal scales.

To test this hypothesis, we analysed whole-brain fMRI data from 100 unrelated subjects from the Human Connectome Project while they performed a cognitivelychallenging two-back task (Barch et al., 2013). Regional BOLD fMRI data were analysed using the same techniques that were applied to the simulated data (i.e., those in Fig. 4), and then independent-samples t-tests were used to contrast between cognitive task engagement and relatively quiescent rest periods. The results of our analysis demonstrated that, when compared to the resting state, task performance was associated with an increase in integration $\left(t=83.8 ; \mathrm{p}=1.02 \times 10^{-93}\right.$; Fig. 5a), a drop in regional diversity $\left(t=29.1 ; p=2.37 \times 10^{-50}\right.$; Fig. $\left.5 b\right)$, increased time-series variability $\left(t=-31.1 ; p=6.83 \times 10^{-53}\right.$; Fig. $\left.5 c\right)$, and less variance explained by the first two principal components $\left(\mathrm{PC}_{1}: \mathrm{t}=5.21 ; \mathrm{p}=1.04 \times 10^{-6} ; \mathrm{PC}_{2}: \mathrm{t}=9.06 ; \mathrm{p}=1.23 \times 10^{-14}\right.$; Fig. 5d).

(a)

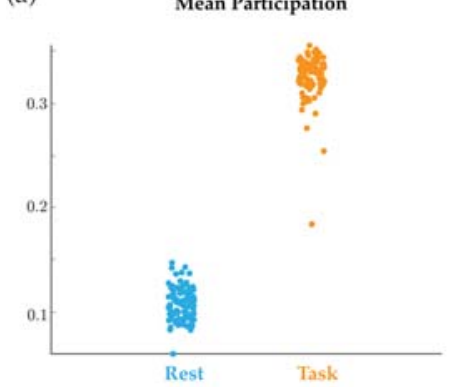

(c)

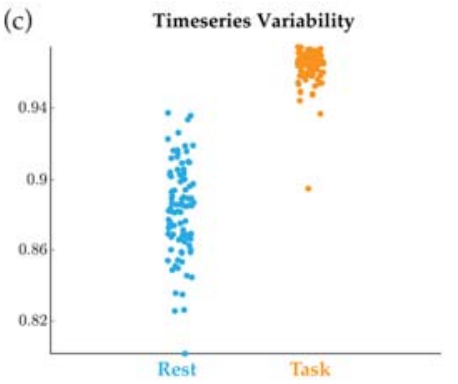

(b)

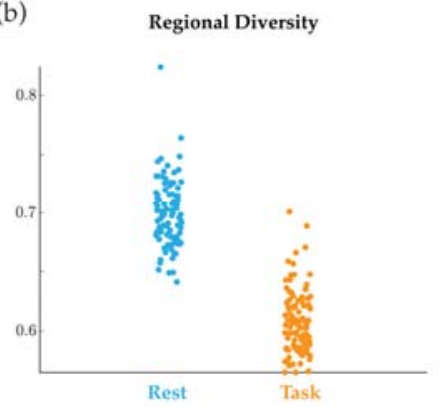

(d)

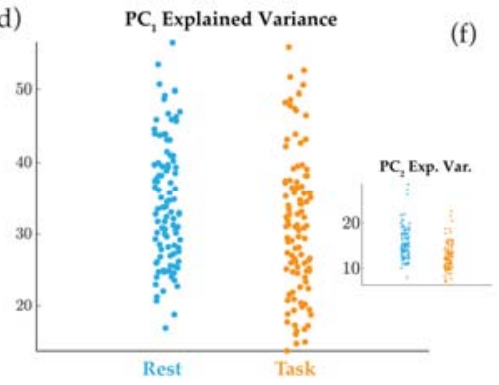

(f)
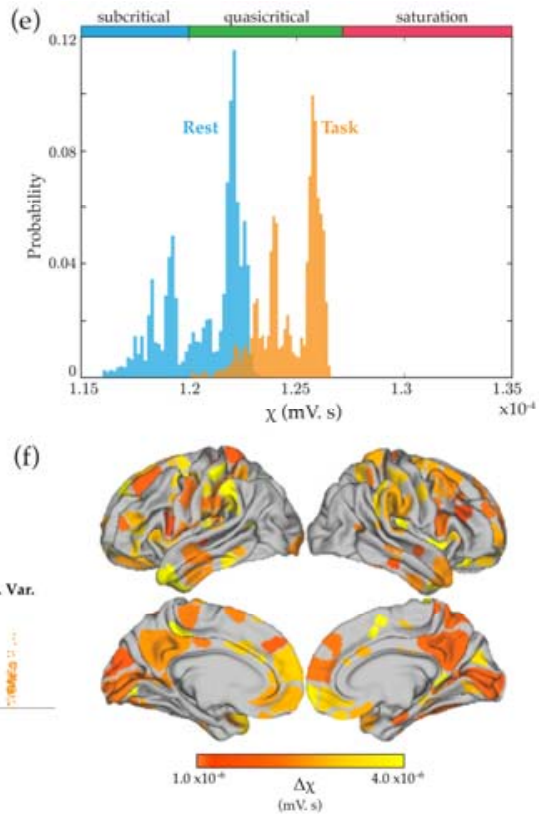

Figure 5 - Signatures of quasi-criticality across task and rest. fMRI data from 100 unrelated subjects during a two-back task from the Human Connectome Project was analysed to determine whether the task and rest states were associated with unique signatures of complex, adaptive brain dynamics. (a) 
Mean Participation was elevated during task performance $\left(t=83.8 ; p=1.02 \times 10^{-93}\right)$; (b) Regional diversity, defined as the variance in the upper triangle of the region-wise functional connectivity matrix, was lower during task performance than rest $\left(t=29.1 ; p=2.37 \times 10^{-50}\right)$; $(c)$ fMRI timeseries variability $\left(t=-31.1 ; p=6.83 \times 10^{-53}\right)$; $(d)$ Variance explained by first principal component $(t=5.21 ; p=$ $\left.1.04 \times 10^{-6}\right) ;\left(d\right.$ - inset) Variance explained by second principal component $\left(t=9.06 ; p=1.23 \times 10^{-14}\right) ;(e)$ Task and rest signatures were applied to a novel stochastic data-fitting algorithm to orient the brain states at different levels of $\chi$ : rest was associated with a lower diffuse coupling ( $\chi^{\text {rest }} 1.22 \pm 0.1 \mathrm{mV} . \mathrm{s}$ ) than task states $\left(\chi^{\text {task }} 1.26 \pm 0.1 \mathrm{mV} . \mathrm{s}\right)$; (f) surface projection of $\Delta \chi$ for each region (Schaefer et al., 2018), generated by independently removing each region of the data, recalculating the signatures, and refitting to generate a $\Delta \chi$.

To orient regional fMRI data onto the corticothalamic model outputs, we created a novel data-fitting approach. Briefly, a cost-function was defined as the difference between the task and rest values for each of the complex network signatures used to analyse systems-wide time series dynamics (Fig. 5a-d). The algorithm then searches for an interval of diffuse coupling, $\Delta \chi$, that minimizes this cost function - that is it finds the $\left[\chi_{1}, \chi_{2}\right]$ that best explains the change in all complex network signatures across task and rest states. Finally, a uniform random walk is performed on the weightings of each metrics gradient to scale its contribution to the overall cost function, effectively mitigating against bias for any one measure in the fitting algorithm. In this way, we were able to estimate the dynamical fingerprint of the underlying state in a manner that was robust to differences in the baseline statistics of each measure.

This approach confirmed that quasi-critical signatures orient rest states to lower levels of diffuse coupling $\left(\chi^{\text {rest }} \sim 1.22 \pm 0.1 \times 10^{-4} \mathrm{mV} \cdot \mathrm{s}\right)$ than those of cognitive task states $\left(\chi^{\text {task }} \sim 1.26 \pm 0.1 \times 10^{-4} \mathrm{mV} \cdot \mathrm{s}\right.$; Fig. 5e). The $\chi$ fit results in a probability distribution (Fig. 5e) since an estimate is made for each new combination of weightings generated per iteration of the algorithm $\left(10^{4}\right)$. The maximum likelihood of the task estimates was found to be coincident with the second peak in regional diversity and proximal to peak integration, suggesting that the brain is balancing flexibility, in the form of high functional diversity, with increased large-scale communication, in the form of network integration.

To aid neuroscientific interpretation, a variation of the group-level model fitting approach was used to provide an estimate of $\Delta \chi$ at the regional-level. To this end, we performed a virtual lesioning of the network (albeit without the benefit of the dimensionality measures, which are calculated across the whole system), in which each of the measures was recalculated following the removal of each node (in turn). 
The algorithm then fits the resultant $\Delta \chi$ which best captured their respective changes, with the notable difference that the upper bound of this range was set to the maximum likelihood of $\chi^{\text {task }}$, so as to ensure each nodes effect was compared to a common baseline (i.e., it finds $\left[\chi_{1}, \chi^{\text {task }}\right]$ ). The $\Delta \chi$ fits were diversely distributed across predominantly frontal and sensory cortex (Fig. 4f), suggesting that diffuse coupling allowed for integration across multiple distinct specialist sub-networks in order to complete the cognitive task. Together, these results confirm the hypothesis that brain activity during task is associated with greater quasi-critical brain dynamics than during rest, and further extend this concept by suggesting a plausible biological mechanism - namely increased diffuse coupling - for these differences.

\section{Discussion}

Here, we used a network of biophysical corticothalamic neural masses, previously fit to human EEG data (Abeysuriya et al., 2015; Robinson et al., 2004), to demonstrate that quasi-critical brain states can be facilitated by the combination of spatially heterogeneous inputs and diffuse network coupling. Gradually increasing diffuse connectivity shifted each region closer to their individually-defined bifurcation, which maximized flexibility (Fig. 2) while also increasing the sensitivity of the network to inputs (Fig. 3b) and system-wide topological integration (Fig. 4a). This constellation of complex network signatures dissociated different cognitive processing modes in empirical brain imaging data (Fig. 5). Together, these results establish a plausible neurobiological implementation of criticality in the brain that is driven by a known neuroanatomical principal. Crucially, the modulation of this physical mechanism (diffuse coupling) is demonstrated to augment flexibility in segregated and integrated operational modes, which in turn are reflected as changes in several key measures of complex adaptive network dynamics.

In previous work, it has been shown that cognitive task performance leads to a more integrated (Shine et al., 2016) and low-dimensional (Shine et al., 2019a) brain state. Here, we demonstrate a simple neuroanatomical principle that may underpin these patterns. Specifically, we showed that, in the presence of the simplest form of spatial heterogeneity (independent noise to each region), increasing diffuse coupling across the network led to the exploitation of multi-stable system dynamics, broadened the systems dynamic repertoire, supported a hierarchy of input response sensitivity and timescales, and maximised temporal flexibility. Indeed, the quasi-critical states that we identified can facilitate functional integration across large spatial and temporal scales through a diverging correlational length, while also retaining the stability of 
the whole system. As such, these mechanisms provide a robust starting point for understanding the evolutionary mechanisms through which the brain learned to augment its functional repertoire across a wide range of scales.

The quasi-critical brain states represented here extend previous ideas on the critical brain hypothesis (Beggs, 2003; Tagliazucchi et al., 2012; Williams-García et al., 2014; Wilting \& Priesemann, 2018), but cast them in a novel, large-scale biophysical brain model. Conceptually, the quasi-critical zone identified in this work represents a state of the system where the dynamic repertoire and flexibility are both maximised. Here, we show that this state can be engaged and disengaged by modulating the impact of diffuse network connectivity. Importantly, this modelling work includes spatial heterogeneities in a minimal form (namely, noise inputs), which permit quasi-critical states while retaining physiologically plausible neural activity. It is also important to note that the quasi-critical state is not a single point but a well-defined region in state space, and thus numerous parameter combinations could be employed by the neurobiology in order to explore this physical niche, which agrees with the extended critical region observed in both human and Caenorhabditis elegans neural networks (Moretti and Muñoz, 2013).

A strength of the approach utilized here is that it relates directly to known characteristics of neuroanatomy. Indeed, there are at least two major systems in the human brain - the ascending arousal system (Samuels and Szabadi, 2008) and the diffuse thalamocortical 'Matrix' projections (Jones, 2001) - that could readily instantiate the diffuse brain signal modelled in our study in a relatively flexible manner. Each of these highly inter-connected (Edlow et al., 2012; Varela, 2014) systems is characterised by relatively diffuse patterns of axonal connectivity that innervate the entire cortical mantle, along with a range of other subcortical, cerebellar and brainstem structures (Jones, 2001; Samuels and Szabadi, 2008). These two systems are also characterized by highly dynamic expression (Aston-Jones and Cohen, 2005; Halassa and Kastner, 2017), suggesting that the relative amount of diffuse coupling may be controlled and shaped as a function of systemic requirements. Despite their relatively broad projection patterns, there is also evidence for more targeted connectivity (Clascá et al., 2012) and segregated processing modes (Totah et al., 2018) within these two systems, which in turn might confer even more precise control over the highly dynamic, distributed neural coalitions that define our waking brain state (Varela et al., 2001). In short, realistic heterogeneity within these systems, such as synaptic, receptor, and cell densities, 
will support the formation of quasi-critical states, and hence the brain may have evolved a way of using quasi-criticality to support distinct operational modes. This would allow low dimensional control over the modes in an energy efficient manner, i.e., functionally partition regions, allocate these to unique features of a task, and then reintegrate their outputs at a later time (Shine et al., 2019a).

The modelling methodology used in this work is distinct from inversion methods, wherein a generative model is fit to data and the resulting parameter estimates are used to elucidate a mechanistic understanding of a phenomenon. Whilst this approach is often informative, it can also result in the over-fitting of parameters. As such, insights from these approaches are difficult to generalize to broader brain states. A complimentary strategy often employed in statistical physics is to define a model according to first principles. While abstract, this strategy affords much greater control over the models' degrees of freedom, and in turn makes any identified results more robust to parameter changes. These two modelling approaches compliment one and other and represent distinct modes of questioning a phenomenon: data-driven (why is the system changing in this way?) vs. hypothesisdriven (i.e., how will the system change if I modify it in this way?). In this work, we utilised a hybrid strategy: we exploited previous data fitting results (Abeysuriya et al., 2015; Robinson et al., 2004) to orient the model in a plausible region of state-space (eyes-closed wakefulness) and then gradually introduced a new feature (diffuse cortical input) while keeping all other parameters constant. The model is thus a predictive framework, in which all of the signatures we identified (Figs. 3 and 4) can be directly attributed to the modulation of diffuse coupling. In addition, by orienting our model in a previously defined state, our outputs can be directly compared with data, though we predict that direct matches to data will require a more realistic structural connectome (Moretti and Muñoz, 2013).

\section{Conclusion}

In summary, we have demonstrated a plausible benefit for the presence of an oftenoverlooked quirk of neuroanatomy: namely, that increases in diffuse coupling can orient the brain in a quasi-critical state that maximizes flexibility, low-dimensionality and a balance between integration and segregation, and that these can explain numerous features of human imaging data. Future work can utilize this model to learn how the balance between targeted and diffuse coupling is disrupted across neurodevelopmental and neurodegenerative disorders. It will also be of major interest to determine how the mechanisms highlighted here interact with the 
complex, heterogeneous patterns of connectivity defined by the white-matter structure of the human connectome, which would likely assign meaningful functional specialization to specific brain regions. 


\section{Materials and Methods}

\section{Corticothalamic neural mass}

The corticothalamic neural mass model used in this work contains four distinct populations: an excitatory pyramidal cell, $e$, and an inhibitory interneuron, $i$, population in the cortex; and an excitatory specific relay nuclei, $s$, and inhibitory thalamic reticular nuclei, $r$, population in the thalamus. The dynamical processes that occur within and between populations in a neural field model are defined as follows:

For each population, the mean soma potential results from incoming postsynaptic potentials (PSPs):

$$
V_{a}(t)=\sum_{b} V_{a b}(t)
$$

where $V_{a b}(t)$ is the result of a postsynaptic potential of type $b$ onto a neuron of type $a$ and $a, b \in\{e, i, r, s\}$. The postsynaptic potential response in the dendrite is given by

$$
D_{a b} V_{a b}(t)=v_{a b} \phi_{a b}\left(t-\tau_{a b}\right)
$$

where the influence of incoming spikes to population $a$ from population $b$ is weighted by a connection strength parameter $v_{a b}=N_{a b} s_{a b}$, with the mean number of connections between the two populations $N_{a b}$ and $s_{a b}$ is the mean strength of response in neuron $a$ to a single spike from neuron $b . \tau_{a b}$ is the average axonal delay for the transmission of signals, and $\phi_{a b}$ is the mean axonal pulse rate from $b$ to $a$.

The operator $D_{a b}$ describes the time evolution of $V_{a b}$ in response to synaptic input,

$$
D_{a b}=\frac{1}{\alpha \beta} \frac{d^{2}}{d t^{2}}+\left(\frac{1}{\alpha}+\frac{1}{\beta}\right) \frac{d}{d t}+1
$$

where $\beta$ and $\alpha$ are the overall rise and decay response rates to the synaptodendritic and soma dynamics.

The mean firing rate of a neural population $Q_{a}(t)$ can be approximately related to its mean membrane potential, $V_{a}(t)$, by 


$$
=\frac{Q_{a}(t)=S_{a}\left[V_{a}(t)\right]}{Q_{a}^{\max }} \frac{\left.\left.\theta_{a}\right\} / \sigma^{\prime}\right]}{1+\exp \left[-\left\{V_{a}(t)-\theta^{\prime}\right.\right.}
$$

which define a sigmoidal mapping function $S_{a}$ with a maximal firing rate $Q_{a}^{\max }$, a mean firing threshold $\theta_{a}$, and a standard deviation of this threshold $\sigma^{\prime} \pi / \sqrt{3}$.

The mean axonal pulse rate is related to the mean firing rate by,

$$
\begin{gathered}
D_{a}(t) \phi_{a}(t)=Q_{a}(t) \\
D_{a}(t)=\frac{1}{\gamma_{a}^{2}} \frac{\partial^{2}}{\partial t^{2}}+\frac{2}{\gamma_{a}} \frac{\partial}{\partial t}+1
\end{gathered}
$$

Here, $\gamma_{a}=v_{a} / r_{a}$ represents the damping rate, where $v_{a}$ is the propagation velocity in axons, and $r_{a}$ is the characteristic axonal length for the population.

Following the approach of previous neural field models, excitatory and inhibitory synapses in the cortex are assumed proportional to the number of neurons (Robinson et al., 2001; Wright and Liley, 1996). This random connectivity approximation results in $v_{e e}=v_{i e}$, and $v_{e i}=v_{i i}$ which implies $V_{e}=V_{i}$ and $Q_{e}=Q_{i}$. Inhibitory population variables can then be expressed in terms of excitatory quantities and are thus not neglected.

The fixed-point attractors, or steady states, of the corticothalamic neural mass are found by setting all time derivatives in the above equations to zero. The steady-state values $\phi_{e}^{(0)}$ of $\phi_{e}$ is then given by solutions of

$$
\begin{gathered}
S^{-1}\left(\phi_{e}^{(0)}\right)-\left(v_{e e}+v_{e i}\right) \phi_{e}^{(0)} \\
=v_{e s} S\left\{v_{s e} \phi_{e}^{(0)}+v_{s r} S\left[v_{r e} \phi_{e}^{(0)}+\frac{v_{r s}}{v_{e s}}\left\{S^{-1}\left(\phi_{e}^{(0)}\right)-\left(v_{e e}+v_{e i}\right) \phi_{e}^{(0)}\right\}\right]+\right. \\
\left.v_{s n} \phi_{n}^{(0)}\right\}
\end{gathered}
$$

where $\phi_{n}^{(0)}$ is the steady state component of the input stimulus (Braitenberg and Schuz, 2013; Wright and Liley, 1996). Roots of Eq. (7) are found using the fzero() function from MATLAB. 
The connection gains between populations, which represent the additional activity generated in postsynaptic nuclei per additional unit input activity from presynaptic nuclei, can be calculated by linearizing Eq. (4) which gives

$$
G_{a b}=\rho_{a} v_{a b}
$$

where

$$
\rho_{a}=\left.\frac{d Q_{a}}{d V_{a}}\right|_{V_{a}^{(0)}}=\frac{\phi_{a}^{(0)}}{\sigma^{\prime}}\left[1-\frac{\phi_{a}^{(0)}}{Q_{a}^{\max }}\right]
$$

It is an important goal of this work to extend the ideas and phenomena already present in an existing biophysical model, which has been compared to human data, instead of a specific model of the phenomena with no bridge towards showing its implementation in the biology.

\begin{tabular}{c|lll} 
Parameter & Description & Value & Unit \\
$\gamma_{e}$ & Cortical damping rate & 116 & $\mathrm{~s}^{-1}$ \\
$Q^{\text {max }}$ & Maximum firing rate & 340 & $\mathrm{~s}^{-1}$ \\
$\theta$ & Firing threshold & 12.9 & $\mathrm{mV}$ \\
$\sigma^{\prime}$ & Threshold spread & 3.8 & $\mathrm{mV}$ \\
$\phi_{n}$ & Input noise amplitude spectral & $1 \times 10^{-5}$ & $\mathrm{~s}^{-1}$ \\
& density & & \\
$\alpha$ & Decay rate of cell-body potential & 83 & $\mathrm{~s}^{-1}$ \\
$\beta$ & Rise rate of cell-body potential & 769 & $\mathrm{~s}^{-1}$ \\
& & & \\
$v_{e e}$ & Intra-node coupling strengths & & $\mathrm{mV} \mathrm{s}$ \\
$v_{e i}$ & & 1.5 & $\mathrm{mV} \mathrm{s}$ \\
$v_{e s}$ & & -3 & $\mathrm{mV} \mathrm{s}$ \\
$v_{s e}$ & & 0.57 & $\mathrm{mV} \mathrm{s}$ \\
$v_{s r}$ & & 3.4 & $\mathrm{mV} \mathrm{s}$ \\
$v_{s n}$ & & -1.5 & $\mathrm{mV} \mathrm{s}$ \\
$v_{r e}$ & & 3.6 & $\mathrm{mV} \mathrm{s}$ \\
$v_{r s}$ & & 0.17 & $\mathrm{mV} \mathrm{s}$ \\
$\tau_{e s}+\tau_{s e}$ & Corticothalamic loop delay & 0.05 & $\mathrm{~ms}$ \\
$v_{e e}^{\text {local }}$ & Local network coupling strength & $1.8 \times 10^{-4}$ & $\mathrm{mV} \mathrm{s}$ \\
$\chi$ & Diffuse network coupling strength & {$[1.15-1.35] \times 10^{-4}$} & $\mathrm{mV} \mathrm{s}$
\end{tabular}


Table 1 - Corticothalamic neural mass parameters. Adapted from (Abeysuriya et al., 2015).

\section{Numerical simulations}

A $12 \times 12$ network of corticothalamic neural masses were simulated using the neural field simulation software, nftSim (Sanz-Leon et al., 2018). The parameters for each neural mass were identically set to "eyes-closed" estimates given in Table 1 (Abeysuriya et al., 2015), which results in simulated activity with a $1 / \mathrm{f}$ spectrum and a peak in the alpha frequency band (8-13 Hz) under moderate network coupling. Each simulation was run for a total of $32 \mathrm{~s}$ with 10 s of initial transients removed using an integration timestep of $\Delta t=2^{-13} \mathrm{~s}$.

\section{Network connectivity and heterogeneity}

The noise terms are individually generated for a node from an identical white noise Gaussian distribution with a mean of $1\left(\mathrm{~s}^{-1}\right)$ and an amplitude spectral density of $10^{-5}$ (arb. units). This serves as the only spatial heterogeneity in the network. The local coupling to each node is a nearest neighbour with diagonal nodes additionally scaled by $1 / \sqrt{2}$. A sweep of the amplitude of these local connections was first performed to determine the location of the ensemble bifurcation (phase transition) point, and then a slightly smaller value was used to ensure the system was proximal to this point but far enough away as to be stable under perturbations from the noise terms. An additional level of network connectivity, called diffuse coupling and represented by the symbol $\chi$, prescribes a given nodes connection to the entire network. This is the only coupling parameter that changes in this work.

\section{Distance to bifurcation}

The network activity incident to each node at a given time point is purely excitatory and as such can be considered as a constant positive post-synaptic potential. In line with this, a constant potential is added to the cortical excitatory population and the steady states of the neural mass are solved numerically. A sweep of this potential change elucidates a saddle-node bifurcation which represents the necessary input, as a first order approximation, required to drive a node to its locally defined critical boundary. The bifurcation point can then be used as a reference for interpreting simulation activity post hoc. That is, at each time point the incident network activity to each node is translated into a distance to bifurcation time series for that target node, which enables parallel analysis of local activity and network induced effects. 
Using this information, we defined three 'working zones': a stable, subcritical zone $(\chi<1.20 \mathrm{mV} \cdot \mathrm{s}$; blue in Fig. 2$)$, where $P_{c}=0$; a quasi-critical zone $(1.20<\chi<1.27$ $\mathrm{mV} \cdot \mathrm{s}$; green in Fig. 2), where $0<P_{c}<100$; and a saturated zone $(\chi>1.27 \mathrm{mV} \cdot \mathrm{s}$; red in Fig. 2), where $P_{c}=100$ in the second half of the simulation. The average population-level firing rate and gain within each zone was used to create an 'ideal' corticothalamic population (Fig. 2). The Pearson's correlation matrix within each zone was then thresholded $(r>0)$, binarized and used to create a force-directed embedding (Fig. 3).

\section{Response to pulse stimulus}

A simulation was first run with no applied pulse stimuli for comparison with stimulus results. Then, $\mathrm{N}=144$ trails were run where a pulse stimulus (amplitude $=$ $1 \mathrm{mV}$; width $=10 \mathrm{~ms}$ ) was applied to a single node at $\mathrm{t}=10 \mathrm{~s}$. The cortical activity from the no-stimuli simulation is subtracted from all pulse trails. Since the noise sequence generated is the same for each trial, this allows a clear mapping of stimuli-induced response. The trials are sorted based on the target nodes average distance to bifurcation within the 8 timepoints pre-stimulus. For visualization purposes, the stimulus-induced response of the targeted node in each trial is averaged across upper, middle, and lower thirds of the sorted distance to bifurcation vector, and the time series is low-pass filtered with a passband frequency of $0.001 \mathrm{~Hz}$. As expected, nodes closest to their bifurcation had the strongest response, and the longest timescale for decaying back to pre-stimulus levels of activity.

\section{Network signatures of criticality}

The time series of the cortical ' $\mathrm{e}$ ' population was used to create a weighted, unthresholded connectivity matrix. A weighted- and signed- version of the Louvain modularity algorithm from the Brain Connectivity Toolbox (Rubinov and Sporns, 2010) was used to iteratively maximizes the modularity statistic, $Q$, for different community assignments until the maximum possible score of $Q$ has been obtained (Equation 4). The modularity estimate for a given network is, therefore, a quantification of the extent to which the network may be subdivided into communities with stronger within-module than between-module connections.

$$
Q_{T}=\frac{1}{v^{+}} \sum_{i j}\left(w_{i j}^{+}-e_{i j}^{+}\right) \delta_{M_{i} M_{j}}-\frac{1}{v^{+}+v^{-}} \sum_{i j}\left(w_{i j}^{-}-e_{i j}^{-}\right) \delta_{M_{i} M_{j}}
$$

where $v$ is the total weight of the network (sum of all negative and positive connections), $w_{i j}$ is the weighted and signed connection between regions $i$ and $j, e_{i j}$ is 
the strength of a connection divided by the total weight of the network, and $\delta_{M i M j}$ is set to 1 when regions are in the same community and 0 otherwise. ' + ' and ' - ' superscripts denote all positive and negative connections, respectively. In our experiment, the $\gamma$ parameter was set to 1.1 (tested within a range of $0.5-2.0$ for consistency across 100 iterations). Given that the community structure of the system changed substantially as a function of $\chi$, a consensus partition was created across the whole range using the 'consensus_und. $m$ ' script from the Brain Connectivity Toolbox.

The participation coefficient quantifies the extent to which a region connects across all modules. This measure has previously been used to characterize diversely connected hub regions within cortical brain networks (e.g., see Power 2013). Here, the Participation Coefficient $(B)$ was calculated for each of the 400 cortical parcels for each subject, where $\kappa_{\text {isT }}$ is the strength of the positive connections of region $i$ to regions in module $s$, and $\kappa_{\mathrm{T}} \mathrm{T}$ is the sum of strengths of all positive connections of region $i$. The participation coefficient of a region is therefore close to 1 if its connections are uniformly distributed among all the modules and 0 if all of its links are within its own module:

$$
B=1-\sum_{s=1}^{n_{M}}\left(\frac{\kappa_{i s T}}{\kappa_{i T}}\right)^{2}
$$

Brain state variability was calculated by taking the standard deviation of the upper triangle of the correlation matrix at each level of $\chi$. Time series variability was estimated using the regional mean of the standard deviation of the cortical ' $\mathrm{e}$ ' population over time. The percentage of explained variance for the top two principal components was calculated by subjecting demeaned cortical ' $\mathrm{e}$ ' population timeseries at each level of $\chi$ to separate principal component analyses.

\section{Whole-brain fMRI analysis}

Minimally pre-processed fMRI data were obtained from 100 unrelated participants (mean age 29.5 years, 55\% female) from the HCP database. For each participant, BOLD data from the left-right encoding session from the N-back task were acquired using multiband gradient echo planar imaging, amounting to $4 \mathrm{~min} 51 \mathrm{sec}$ of data (405 individual TRs) per subject. Pre-processed (Shine et al., 2016, 2019a) but temporally unfiltered data was extracted from 333 cortical parcels (Gordon et al., 2016). The time points associated with each cognitively-challenging task-blocks and the interspersed rest blocks were convolved with a canonical haemodynamic response function (using the spm_hrf.m function from SPM12). 
To estimate functional connectivity between the 333 cortical ROIs, we used the Multiplication of Temporal Derivatives ( $M$ ) technique (Shine et al., 2015). $M$ is computed by calculating the point-wise product of temporal derivative of pairwise time series (Equation 1). The resultant score is then averaged over a temporal window, $w$, in order to reduce the contamination of high-frequency noise in the time-resolved connectivity data. A window length of 20 TRs was used in this study, though results were consistent across a range of $w$ values (10-50 TRs). To ensure relatively smooth transitions between each task, connectivity analyses were performed on each individual task separately, and were subsequently concatenated. In addition, all analyses involving connectivity (or the resultant topological estimates) incorporated the junction between each task as a nuisance regressor.

$$
M_{i j t}=\frac{1}{w} \sum_{t}^{t+w} \frac{\left(t_{\left.i t^{\prime} \times t_{j t^{\prime}}\right)}\right.}{\left(\sigma_{t_{i}} \times \sigma_{t_{j}}{ }^{\prime}\right)}
$$

Where for each time point, $t$, the $M$ for the pairwise interaction between region $i$ and $j$ is defined according to equation 1 , where $t^{\prime}$ is the first temporal derivative $(t+1-t)$ of the $i^{\text {th }}$ or $j^{\text {th }}$ time series at time $t, \sigma$ is the standard deviation of the temporal derivative time series for region $i$ or $j$ and $w$ is the window length of the simple moving average. This equation can then be calculated over the course of a time series to obtain an estimate of time-resolved connectivity between pairs of regions. Timeresolved values of $B_{T}$ are then calculated on each weighted, signed connectivity matrix. Values of each measure were compared statistically using a series of nonparametric permutation tests (Nichols and Holmes, 2003) in which the group identity (i.e., rest vs. task) was randomly shuffled in order to populate a null distribution (5,000 iterations).

\section{Gradient fitting the model to whole-brain fMRI data}

Firstly, participation, regional diversity, time-series variability and variance explained by the first two principal components are calculated on the whole-brain imaging data and the model outputs for each value of diffuse coupling. Since the absolute values of these measures do not form a fair point of comparison with outputs from our simplified corticothalamic model, we focus on their relative differences across task and rest (i.e., what interval of diffuse coupling makes the most sense of the metric changes). Thus, for each measure the difference between rest and task is calculated to form 5 gradients that are fit to the corresponding 
gradients of the model outputs across levels of diffuse coupling. This is done by subsampling the model outputs (Figs 4 (a), (b), and (c)) at progressively coarser steps sizes, calculating the gradient numerically using diff() function from MATLAB, and then finding the $x$ value (which is a subsample interval) that minimizes the cost function. The final estimate is the average across all subsampling scales. Finally, in order to mitigate against bias for any one metric in the fit, a uniform random walk is performed on the $0-1$ weightings of each gradient metric to scale its contribution to the cost function.

Two distinct approaches are used for the whole-brain and regional estimates, respectively. For the whole-brain estimates, the algorithm is free to change the upper and lower bounds of the diffuse coupling interval $\chi_{1}, \chi_{2}$. For the regional estimates, we use the maximum likelihood from the task estimate of diffuse coupling $\chi^{\text {task }} \sim 1.26$ $\pm 0.1 \times 10-4 \mathrm{mV} \cdot \mathrm{s}$ as the upper bound for the search, and thus only the lower bound is free to change $\left[\chi_{1}, \chi^{\text {task }}\right]$. A virtual lesioning approach is then used, where each node is removed from the data (only a single node is ever removed at a time) and the algorithm is run to estimate the new diffuse value (relative to the task estimate). The result is an estimate of the change in diffuse coupling facilitated by each node in the network. 


\section{References}

Abeysuriya, R.G., Rennie, C.J., and Robinson, P.A. (2015). Physiologically based arousal state estimation and dynamics. J. Neurosci. Methods 253, 55-69.

Aertsen, A., Diesmann, M., and Gewaltig, M.O. (1996). Propagation of synchronous spiking activity in feedforward neural networks. J. Physiol. Paris 90, 243-247. Aston-Jones, G., and Cohen, J.D. (2005). AN INTEGRATIVE THEORY OF LOCUS COERULEUS-NOREPINEPHRINE FUNCTION: Adaptive Gain and Optimal Performance. Annu. Rev. Neurosci. 28, 403-450.

Barch, D.M., Burgess, G.C., Harms, M.P., Petersen, S.E., Schlaggar, B.L., Corbetta, M., Glasser, M.F., Curtiss, S., Dixit, S., Feldt, C., et al. (2013). Function in the human connectome: Task-fMRI and individual differences in behavior. Neuroimage 80, 169189.

Bojak, I., and Liley, D.T.J. (2005). Modeling the effects of anesthesia on the electroencephalogram. Phys. Rev. E - Stat. Nonlinear, Soft Matter Phys. 71, 1-22. Braitenberg, V., and Schuz, A. (2013). Cortex: Statistics and Geometry of Neuronal Connectivity (Berlin).

Breakspear, M. (2017). Dynamic models of large-scale brain activity. Nat. Neurosci. 20, 340-352.

Breakspear, M., Roberts, J.A., Terry, J.R., Rodrigues, S., Mahant, N., and Robinson, P.A. (2006). A unifying explanation of primary generalized seizures through nonlinear brain modeling and bifurcation analysis. Cereb. Cortex 16, 1296-1313. Bullmore, E., and Sporns, O. (2012). The economy of brain network organization. Nat. Rev. Neurosci. 13, 336-349.

Clascá, F., Rubio-Garrido, P., and Jabaudon, D. (2012). Unveiling the diversity of thalamocortical neuron subtypes. Eur. J. Neurosci. 35, 1524-1532.

Cocchi, L., Sale, M. V., Gollo, L.L., Bell, P.T., Nguyen, V.T., Zalesky, A., Breakspear, M., and Mattingley, J.B. (2016). A hierarchy of timescales explains distinct effects of local inhibition of primary visual cortex and frontal eye fields. Elife 5, 1-17.

Cocchi, L., Gollo, L.L., Zalesky, A., and Breakspear, M. (2017). Criticality in the brain: A synthesis of neurobiology, models and cognition. Prog. Neurobiol. 158, 132-152. Cohen, J.R., and D'Esposito, M. (2016). The segregation and integration of distinct brain networks and their relationship to cognition. J. Neurosci. 36, 12083-12094. Cunningham, J.P., and Yu, B.M. (2014). Dimensionality reduction for large-scale neural recordings. Nat. Neurosci. 17, 1500-1509.

David Servan-Schreiber, H.P. and J.D.C. (1990). A Network Model of Catecholamine Effects: Gain, Signal-to-Noise Ratio, and Behavior. J. Clin. Endocrinol. Metab. 4971, 892-895.

Deco, G., and Jirsa, V.K. (2012). Ongoing Cortical Activity at Rest: Criticality, Multistability, and Ghost Attractors. J. Neurosci. 32, 3366-3375.

Deco, G., Jirsa, V.K., Robinson, P.A., Breakspear, M., and Friston, K. (2008). The dynamic brain: From spiking neurons to neural masses and cortical fields. PLoS 
Comput. Biol. 4.

Edlow, B.L., Takahashi, E., Wu, O., Benner, T., Dai, G., Bu, L., Grant, P.E., Greer, D.M., Greenberg, S.M., Kinney, H.C., et al. (2012). Neuroanatomic Connectivity of the Human Ascending Arousal System Critical to Consciousness and Its Disorders. J. Neuropathol. Exp. Neurol. 71, 531-546.

Fontenele, A., Vasconcelos, N.A.P. de, Feliciano, T., Aguiar, L., Soares-Cunha, C., Coimbra, B., Porta, L.D., Ribeiro, S., Rodrigues, A.J., Sousa, N., et al. (2018).

Criticality between cortical states. Phys. Rev. Lett. 122, 454934.

Gordon, E.M., Laumann, T.O., Adeyemo, B., Huckins, J.F., Kelley, W.M., and Petersen, S.E. (2016). Generation and Evaluation of a Cortical Area Parcellation from Resting-State Correlations. Cereb. Cortex 26, 288-303.

Halassa, M.M., and Kastner, S. (2017). Thalamic functions in distributed cognitive control. Nat. Neurosci. 20, 1669-1679.

Hearne, L.J., Cocchi, L., Zalesky, A., and Mattingley, J.B. (2017). Reconfiguration of brain network architectures between resting-state and complexity-dependent cognitive reasoning. J. Neurosci. 37, 8399-8411.

Honey, C.J., Thesen, T., Donner, T.H., Silbert, L.J., Carlson, C.E., Devinsky, O., Doyle, W.K., Rubin, N., Heeger, D.J., and Hasson, U. (2012). Slow Cortical Dynamics and the Accumulation of Information over Long Timescales. Neuron 76, 423-434. Jirsa, V.K., and Haken, H. (1996). Field theory of electromagnetic brain activity. Phys. Rev. Lett. 77, 960-963.

Jones, E.G. (2001). The thalamic matrix and thalamocortical synchrony. Trends Neurosci. 24, 595-601.

Jones, E.G. (2002). Thalamic circuitry and thalamocortical synchrony. Philos. Trans. R. Soc. B Biol. Sci. 357, 1659-1673.

Kehagia, A.A., Murray, G.K., and Robbins, T.W. (2010). Learning and cognitive flexibility: Frontostriatal function and monoaminergic modulation. Curr. Opin. Neurobiol. 20, 199-204.

Kinouchi, O., and Copelli, M. (2006). Optimal dynamical range of excitable networks at criticality. Nat. Phys. 2, 348-352.

McIntosh, A.R., and Jirsa, V.K. (2019). The hidden repertoire of brain dynamics and dysfunction. Netw. Neurosci. 3, 994-1008.

Miller, P. (2016). Dynamical systems, attractors, and neural circuits [version 1; referees: 3 approved]. F1000Research 5, 1-18.

Moretti, P., and Muñoz, M.A. (2013). Griffiths phases and the stretching of criticality in brain networks. Nat. Commun. 4.

Müller, E.J., van Albada, S.J., Kim, J.W., and Robinson, P.A. (2017). Unified neural field theory of brain dynamics underlying oscillations in Parkinson's disease and generalized epilepsies. J. Theor. Biol. 428.

Muñoz, M.A. (2018). Colloquium: Criticality and dynamical scaling in living systems. Rev. Mod. Phys. 90, 31001.

Nichols, T., and Holmes, A. (2003). Nonparametric Permutation Tests for Functional Neuroimaging. Hum. Brain Funct. Second Ed. 25, 887-910. 
Nunez, P.L. (1974). The brain wave equation: a model for the EEG. Math. Biosci. 21, 279-297.

Rennie, C.J., Robinson, P.A., and Wright, J.J. (1999). Effects of local feedback on dispersion of electrical waves in the cerebral cortex. Phys. Rev. E - Stat. Physics, Plasmas, Fluids, Relat. Interdiscip. Top. 59, 3320-3329.

Roberts, J.A., and Robinson, P.A. (2008). Modeling absence seizure dynamics: Implications for basic mechanisms and measurement of thalamocortical and corticothalamic latencies. J. Theor. Biol. 253, 189-201.

Robinson, P.A., Rennie, C.J., and Wright, J.J. (1997). Propagation and stability of waves of electrical activity in the cerebral cortex. Phys. Rev. E - Stat. Physics, Plasmas, Fluids, Relat. Interdiscip. Top. 56, 826-840.

Robinson, P.A., Rennie, C.J., Wright, J.J., and Bourke, P.D. (1998). Steady states and global dynamics of electrical activity in the cerebral cortex. Phys. Rev. E - Stat. Physics, Plasmas, Fluids, Relat. Interdiscip. Top. 58, 3557-3571.

Robinson, P.A., Rennie, C.J., Wright, J.J., Bahiumuli, H., Gordon, E., and Rowe, D.L. (2001). Prediction of electrocnccphulographic spectra from neurophysiology. Phys. Rev. E - Stat. Nonlinear, Soft Matter Phys. 63, 0219031-02190318.

Robinson, P.A., Rennie, C.J., and Rowe, D.L. (2002). Dynamics of large-scale brain activity in normal arousal states and epileptic seizures. Phys. Rev. E - Stat. Physics, Plasmas, Fluids, Relat. Interdiscip. Top. 65, 9.

Robinson, P.A., Rennie, C.J., Rowe, D.L., and O'Connor, C. (2004). Estimation of multiscale neurophysiologic parameters by electroencephalographic means. Hum. Brain Mapp. 23, 53-72.

Robinson, P.A., Rennie, C.J., Rowe, D.L., O'Connor, S.C., and Gordon, E. (2005). Multiscale brain modelling. Philos. Trans. R. Soc. B Biol. Sci. 360, 1043-1050. Rubinov, M., and Sporns, O. (2010). Complex network measures of brain connectivity: Uses and interpretations. Neuroimage 52, 1059-1069.

Samuels, E., and Szabadi, E. (2008). Functional Neuroanatomy of the Noradrenergic Locus Coeruleus: Its Roles in the Regulation of Arousal and Autonomic Function Part II: Physiological and Pharmacological Manipulations and Pathological Alterations of Locus Coeruleus Activity in Humans. Curr. Neuropharmacol. 6, 254285.

Sanz-Leon, P., Robinson, P.A., Knock, S.A., Drysdale, P.M., Abeysuriya, R.G., Fung, F.K., Rennie, C.J., and Zhao, X. (2018). NFTsim: Theory and Simulation of Multiscale Neural Field Dynamics.

Schaefer, A., Kong, R., Gordon, E.M., Laumann, T.O., Zuo, X.-N., Holmes, A.J., Eickhoff, S.B., and Yeo, B.T.T. (2018). Local-Global Parcellation of the Human Cerebral Cortex from Intrinsic Functional Connectivity MRI. Cereb. Cortex 28, 30953114.

Shine, J.M. (2019). Neuromodulatory Influences on Integration and Segregation in the Brain. Trends Cogn. Sci. 23, 572-583.

Shine, J.M., Koyejo, O., Bell, P.T., Gorgolewski, K.J., Gilat, M., and Poldrack, R.A. (2015). Estimation of dynamic functional connectivity using Multiplication of 
Temporal Derivatives. Neuroimage 122, 399-407.

Shine, J.M., Bissett, P.G., Bell, P.T., Koyejo, O., Balsters, J.H., Gorgolewski, K.J., Moodie, C.A., and Poldrack, R.A. (2016). The Dynamics of Functional Brain Networks: Integrated Network States during Cognitive Task Performance. Neuron 92, 544-554.

Shine, J.M., Aburn, M.J., Breakspear, M., and Poldrack, R.A. (2018). The modulation of neural gain facilitates a transition between functional segregation and integration in the brain. Elife 7, 1-16.

Shine, J.M., Breakspear, M., Bell, P.T., Ehgoetz Martens, K., Shine, R., Koyejo, O., Sporns, O., and Poldrack, R.A. (2019a). Human cognition involves the dynamic integration of neural activity and neuromodulatory systems. Nat. Neurosci. 22, 289296.

Shine, J.M., Hearne, L.J., Breakspear, M., Poldrack, R.A., Mattingley, J.B., and Cocchi, L. (2019b). The Low-Dimensional Neural Architecture of Cognitive Complexity Is Related to Activity in Medial Thalamic Nuclei Report The Low-Dimensional Neural Architecture of Cognitive Complexity Is Related to Activity in Medial Thalamic Nuclei. 1-7.

Sporns, O., Tononi, G., and Edelman, G.M. (2000). Connectivity and complexity: The relationship between neuroanatomy and brain dynamics. Neural Networks 13, 909922.

Steyn-Ross, M.L., Steyn-Ross, D.A., and Sleigh, J.W. (2004). Modelling general anaesthesia as a first-order phase transition in the cortex. Prog. Biophys. Mol. Biol. 85, 369-385.

Tagliazucchi, E., Balenzuela, P., Fraiman, D., and Chialvo, D.R. (2012). Criticality in large-scale brain fmri dynamics unveiled by a novel point process analysis. Front. Physiol. 3 FEB, 1-12.

Totah, N.K., Neves, R.M., Panzeri, S., Logothetis, N.K., and Eschenko, O. (2018). The Locus Coeruleus Is a Complex and Differentiated Neuromodulatory System. Neuron 99, 1055-1068.e6.

Varela, C. (2014). Thalamic neuromodulation and its implications for executive networks. Front. Neural Circuits 8, 1-22.

Varela, F., Lachaux, J.P., Rodriguez, E., and Martinerie, J. (2001). The brainweb: Phase synchronization and large-scale integration. Nat. Rev. Neurosci. 2, 229-239. Vojta, T. (2006). Rare region effects at classical, quantum and nonequilibrium phase transitions. J. Phys. A. Math. Gen. 39.

Williams-García, R. V., Moore, M., Beggs, J.M., and Ortiz, G. (2014). Quasicritical brain dynamics on a nonequilibrium Widom line. Phys. Rev. E - Stat. Nonlinear, Soft Matter Phys. 90, 1-8.

Wilson, H.R., and Cowan, J.D. (1973). A mathematical theory of the functional dynamics of cortical and thalamic nervous tissue. Kybernetik 13, 55-80.

Wilting, J., and Priesemann, V. (2018). Inferring collective dynamical states from widely unobserved systems. Nat. Commun. 9.

Wright, J.J., and Liley, D.T.J. (1996). Dynamics of the brain at global and microscopic 
scales: Neural networks and the EEG. Behav. Brain Sci. 19, 285-295.

\section{Supplementary material}

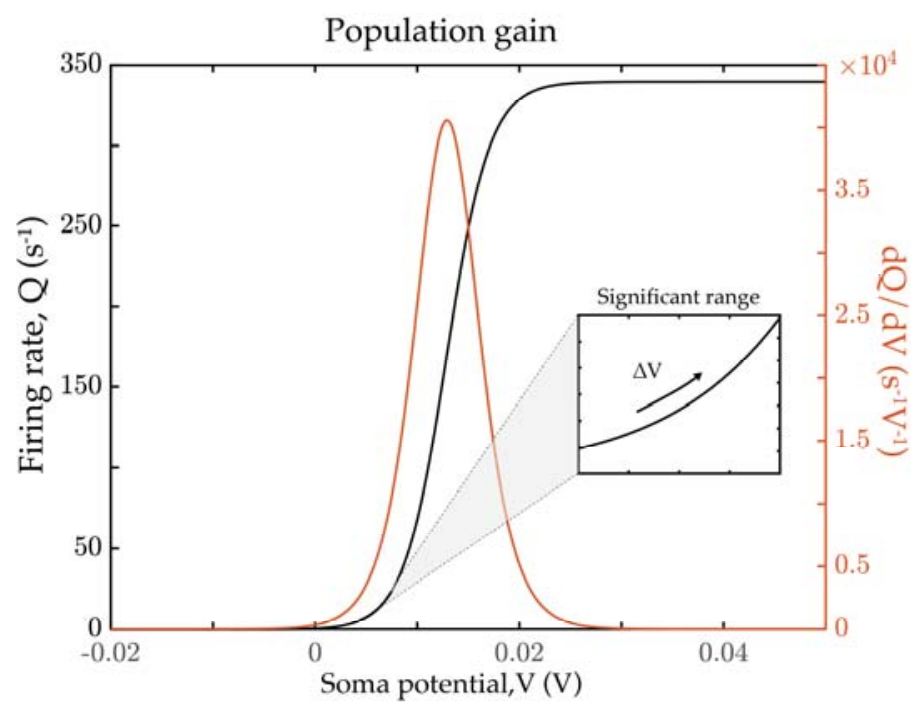

Figure S1 -Population firing rate gain. (left: black) sigmoidal mapping of firing rate to soma potential; (right: orange) first derivative of the sigmoid function. The inset shows the range of postsynaptic potentials significant for the steady-state population firing rates as a function of induced postsynaptic potential. Network coupling in this model is facilitated by connecting cortical excitatory populations between each node. This means that as diffuse coupling increases, the network-induced positive postsynaptic potential generated increases in the receiving nodes cortical population. The effect of this increase can be seen in Fig. S1a where a sweep of constant postsynaptic potential shows the deformation of steady state firing rate attractors. At $\sim 0.12 \mathrm{mV}$, the low attractor is lost through a saddle-node bifurcation leaving only the high firing attractor. Notably, the relative difference of the thalamic reticular nuclei (TRN) and specific relay nuclei (SRN) firing rate attractors is inverted either side of the bifurcation. The simulated time series of cortical firing rates, given in Fig. S1 (b), can then be understood as a 'smearing' of the results in Fig. S1a. 

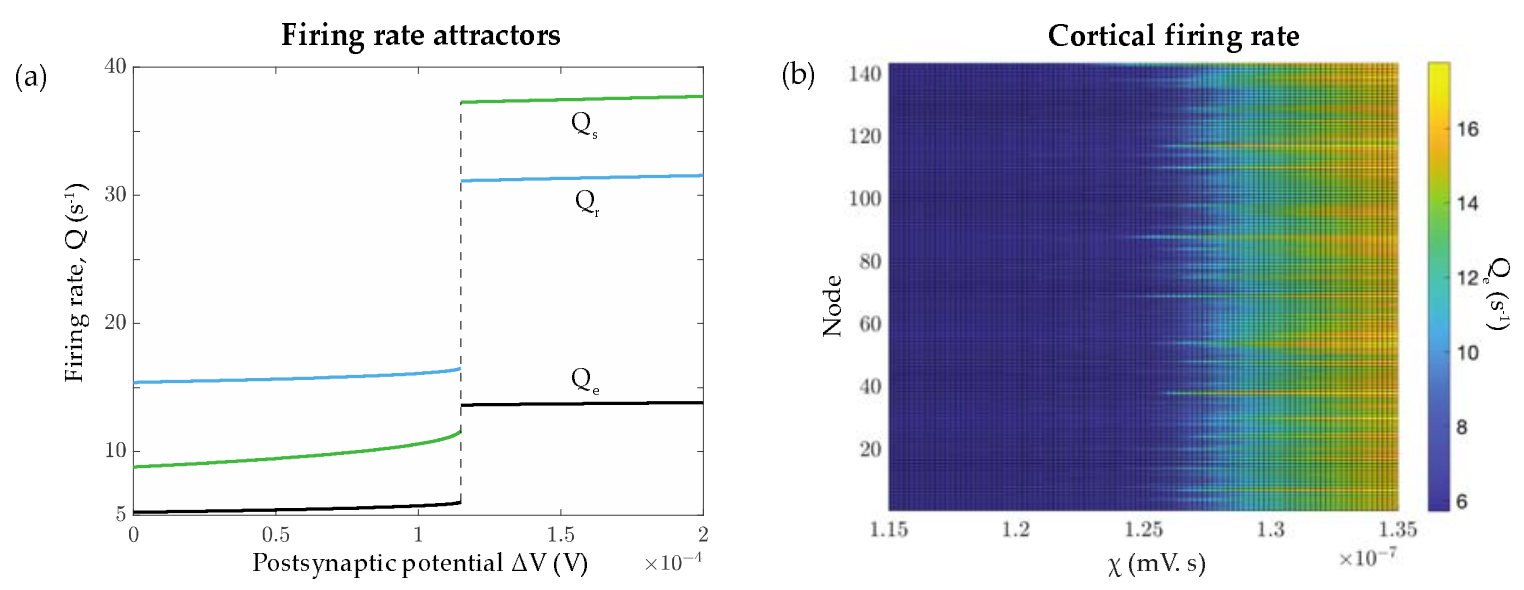

Figure S2- Corticothalamic firing rate attractors across the critical point. (a) Steady-state population firing rates as a function of induced postsynaptic potential. (Qe - cortical excitatory nuclei, Qs thalamic specific relay nuclei, Qr - thalamic reticular nucleus). (b) Simulated mean cortical firing rate for each node as a function of diffuse coupling. 
(a)

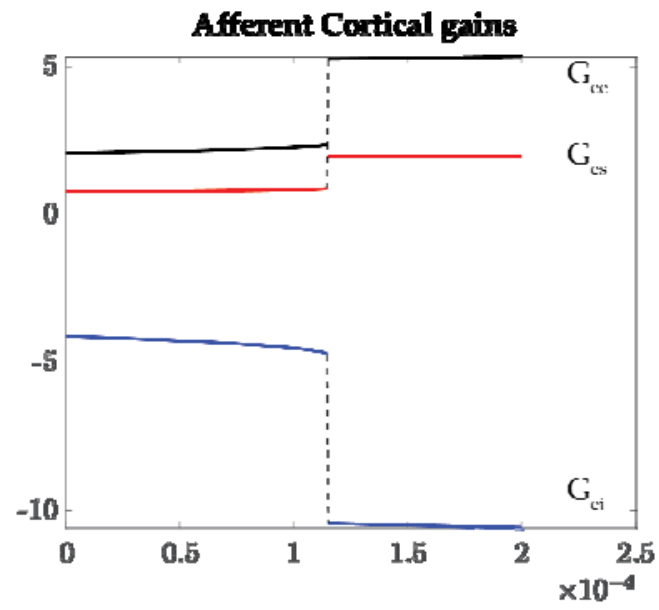

(c)

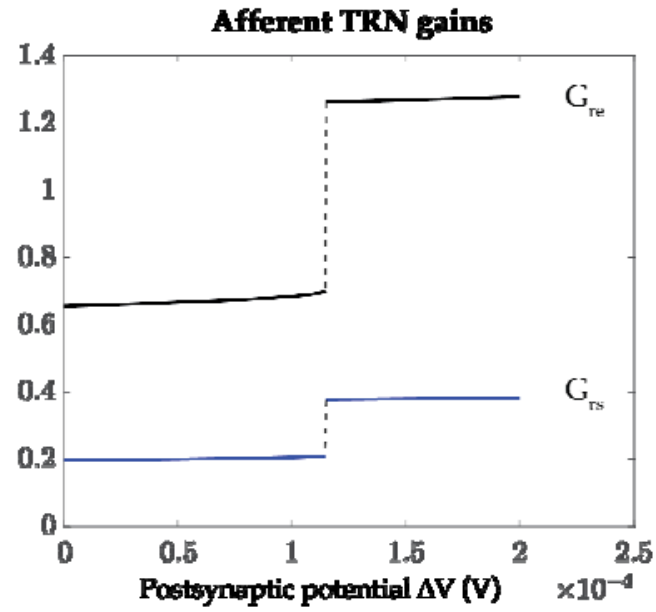

(b)

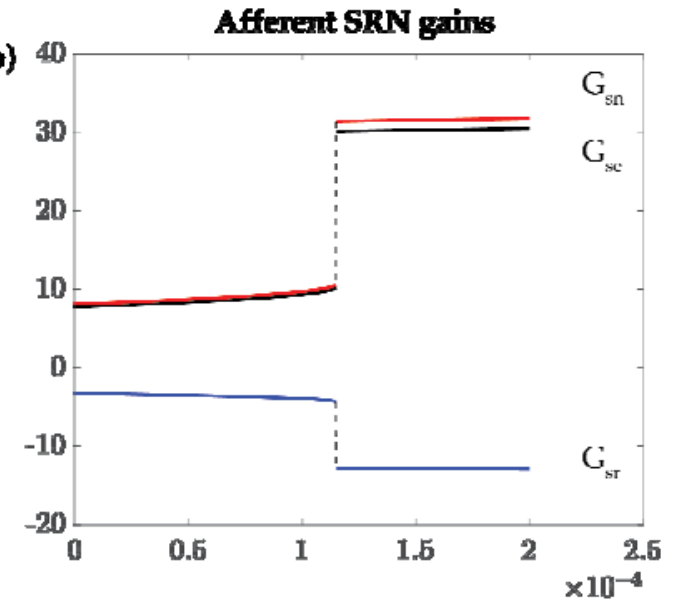

(d)

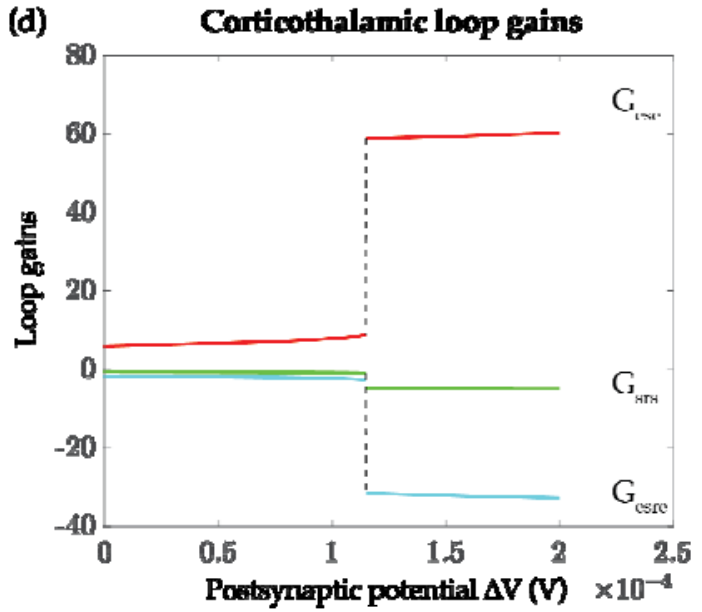

Figure S3 - Corticothalamic steady state gains across the critical point. (a) Excitatory cortical input gains from the excitatory (G_ee), and inhibitory (G_ei) cortex, and the SRN (G_es). (b) SRN thalamic input gains from the cortex (G_se), TRN (G_sr), and external stimuli (G_sn). (c) TRN thalamic input gains from the cortex (G_re), and SRN (G_rs). (d) Cortico-thalamo-cortical loop gains as a function of induced postsynaptic potential. The intra-node corticothalamic gains are given in Fig. S2. As the system crosses the bifurcation point, both the TRN and SRN show a strengthened response to all inputs. In addition, the cortico-SRN-cortical loop gain, which resonates to given the characteristic alpha $(\sim 10 \mathrm{~Hz})$ oscillation in wake, remains dominant. 